\title{
CRESST Detectors for Nonbaryonic Cold Dark Matter Particles
}

\author{
Thomas Jagemann on behalf of the CRESST Collaboration \\ Physik-Department E15, Technische Universität München, \\ James-Franck-Straße, 85748 Garching, Germany
}

\begin{abstract}
The CRESST experiment ${ }^{1}$ is set up for the direct detection of Weakly Interacting Massive Particles (WIMPs) which our Galactic dark matter halo possibly consists of. The employed detection method is elastic scattering by nuclei. The recoiling nucleus deposits most of its energy in the form of lattice vibrations in the detector. Cooling the detector to very low temperatures $(\mathrm{mK})$ enhances the temperature rise due to the energy deposition. The crucial parameter for direct WIMP searches is the sensitivity to the WIMP interaction cross section in a certain range of possible WIMP masses. CRESST is now sensitive enough to explore the parameter space predicted by supersymmetric models.
\end{abstract}

\section{Motivation and Candidates}

Dark matter candidates accounting for the galactic halo should fulfill the requirement to be stable, only weakly interacting and should explain both the galactic rotational curves and structure formation in the early universe. Theoretical elementary particle physics offers several hypothetical candidates for dark matter. The WIMP for example is usually discussed in the framework of Minimal Supersymmetric Models, providing a well motivated candidate through the lightest supersymmetric particle called a "neutralino".

If WIMPS solve the dark matter problem, they are gravitationally bound to our Galaxy. Usually it is assumed that they obey a Maxwellian velocity distribution in the Galactic rest frame with a root mean square velocity of $270 \mathrm{~km} \mathrm{~s}^{-1}$ and cut-off at an escape velocity of $650 \mathrm{~km} \mathrm{~s}^{-1}$. At the Galactic distance of the solar system their mass density is expected to be $0.3 \mathrm{GeV} \mathrm{cm}^{-3}$.

\section{Principle of Detection}

WIMPs are expected to interact with ordinary matter by elastic scattering by nuclei and all direct detection schemes have focused on this possibility. Recoil energies of WIMPs in the mentioned mass range would deposit up to $50 \mathrm{keV}$ with a low event rate smaller than 1 count $/ \mathrm{keV} /$ day $/ \mathrm{kg}$ target mass. Direct

\footnotetext{
${ }^{1}$ Collaboration partners are: Max-Planck-Institut für Physik, Munich, Germany; University of Oxford, Oxford, United Kingdom; and Laboratori Nazionali del Gran Sasso (LNGS), Assergi, Italy
} 
detection methods therefore need to be highly sensitive to nuclear recoils at a low energy threshold, while an extremely low radioactive background must be provided for.

Conventional methods for direct detection rely on the ionization or scintillation caused by the recoiling nucleus. On the other hand, such recoils have a low ionization and scintillation efficiency. Since the principal effect of a WIMP nuclear recoil is the generation of lattice vibrations (phonons) with much lower energy excitations, a detector capable of measuring these phonons must be of superior sensitivity. A temperature rise due to the absorption of phonons will be higher at lower operating temperatures $(\Delta T \sim E / C)$ as the heat capacity decreases. Thus, by operating the detector at very low temperatures $(\sim 20 \mathrm{mK})$, a low energy threshold combined with a high energy resolution is achieved.

\section{First Results}

The analysis of dark matter limits for the two possible WIMP-nucleon cross sections, i.e. spin-dependent and spin-independent interaction, searches for the cross section which is just large enough that the amount of WIMP events becomes inconsistent with the measured spectrum at a given confidence level (here $90 \% \mathrm{CL}$ ). After calculating the shape of the energy spectrum for a given WIMP mass, $\sigma_{0}$ is determined by a maximum-likelihood comparison of the calculated WIMP spectrum with the measured energy spectrum. The analysis is aiming to find the amount of WIMP events that is just too large to be hidden under the measured spectrum.
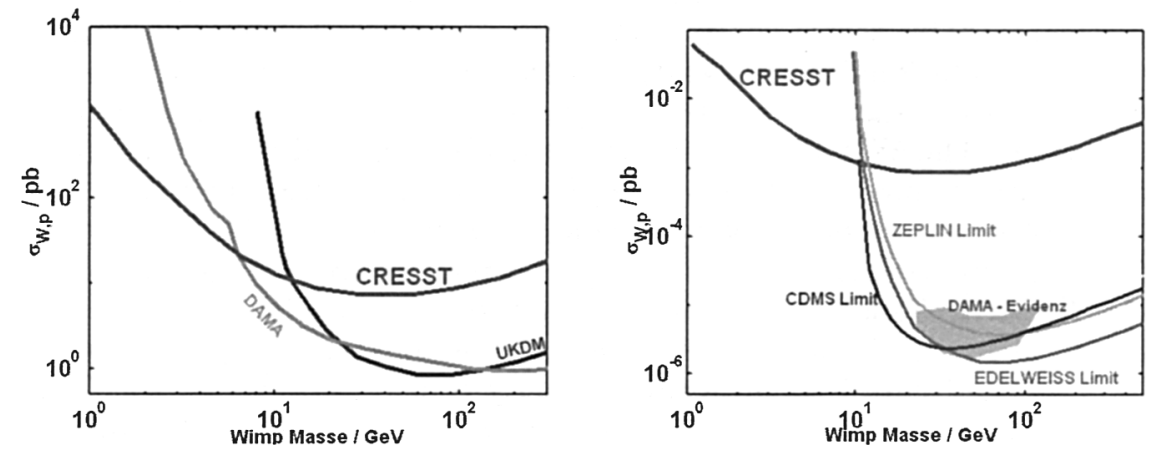

Figure 1. Left: Spin-dependent interaction. Right: Coherent interaction. (Angloher et al. 2002)

\section{References}

Angloher, G., 2002, on behalf of the CRESST Collaboration, in Astrop. Physics $18,43$. 